\title{
Distribution and Diversity of Macrobenthos in Different Mangrove Ecosystems of Tamil Nadu Coast, India
}

\author{
Thilagavathi B*, Varadharajan D, Babu A, Manoharan J, Vijayalakshmi S and Balasubramanian T \\ Faculty of Marine Sciences, Centre of Advanced Study in Marine Biology, Annamalai University, Parangipettai-608 502, Tamil Nadu, India
}

\begin{abstract}
This paper deals with the spatial distribution and diversity of macrobenthos and their relationships between physico-chemical parameters of the water and sediment in different mangrove habitats of Tamil Nadu, India during different seasons of the year-2011. Among the different ecosystems of mangrove benthic faunal assemblages, macrofauna density, richness, evenness and Shannon-wiener index were the highest and the Simpson dominance index was medial at riverine mangrove community. However, the Pielou Evenness index of riverine mangrove community was slightly lower than other communities. The similarities among the macrobenthic communities at different sampling sites were determined using Bray-Curtis similarity coefficient and ordinations of non-metric multidimensional scaling (MDS). One hundred fifty six species were recorded in developing (102 polychaetes, 10 bivalves, 11 gastropods, 24 amphipods, 6 isopods and 3 cumacea), two hundred fifty two species were recorded in riverine (151 polychaetes, 12 bivalves, 16 gastropods, 53 amphipods, 16 isopods and 4 cumacea) and one hundred sixty three species were recorded in island mangrove ecosystem (105 polychaetes, 10 bivalves, 16 gastropods, 21 amphipods, 9 isopods and 2 cumacea). Among the three ecosystems, a total of 292 benthic macrofauna consisting of 188 species of polychaetes, 12 species of bivalves, 17 species of gastropods, 55 species of amphipods, 16 species of isopods and 4 species of cumacea were recorded. However, there were obvious differences among the community structures in the three mangrove habitats. This result implied that the different mangrove ecosystem had different effects on the macrofauna communities and shed light on the macrofauna adaptation capability to specific habitats.
\end{abstract}

Keywords: Mangrove ecosystem; Biodiversity; Macro fauna; Physico-chemical; Seasonal variation

\section{Introduction}

Plants ecosystems are a habitat for a wide variety of species, some occurring in high densities and provide food and shelter for a large number of commercially valuable finfish and shellfishes. They are productivehabitatsand support coastal fisheries [1]. Themangroveforests are extremely important coastal resources, which are vital for socioeconomic development of the region. As a detritus-based ecosystem, leaf litter from the mangroves provides the basis for adjacent aquatic and terrestrial food webs. It also serves as breeding, feeding, and nursery grounds for most of the commercially important finfish and shellfishes, on which thousands of coastal people depend for their livelihood. It is considered to have physical, chemical, and biological processes which promote the adaptation of inhabiting organisms to tolerate greater amplitude of environmental characters both morphologically and physiologically. Krom MD and Berner RA [2] have reported that the decomposition of organic matter consists of nutrients such as nitrogen and phosphorus, which play a vital role in the establishment of healthy mangroves. However, sediment where the animals inhabit often acts as buffer either as a source or sink of nutrients especially phosphorus by adsorption and desorption reactions [2,3]. Hence, the sediment plays a crucial role on benthic faunal diversity in the mangrove ecosystem. Benthic organisms constitute an important component that influences the productivity of the habitat to a greater extent. Benthos helps in the recycling of nutrients, which in turn promotes primary productivity. A detailed and complete knowledge of the bottom fauna is not only important for the determination of productivity [4] but is also helpful in understanding the diversity of the habitat. Macrofauna are the most widely studied benthic organisms which are retained on $0.5 \mathrm{~mm}$ sieve. They reside beneath the sediment surface in burrows and tubes. Thus, seemingly, the bottom of the mangrove substratum habitats forms and a wide array of macrobenthic organisms of various size and taxonomic categories. Indian mangrove ecosystems are known to have a total of 3,985 biological species that include 919 floral species and 3,066 faunal species. Of the biological species, the faunal species occupy about $77 \%$, and the floral species $23 \%$. Thus, the faunal species component is about three times greater than the floral component of the mangrove ecosystem [5,6]. Of these, polychaetes, molluscs, and crustaceans are found to be the major macrobenthic organisms in mangrove environment. Most of the macrobenthos assist in the breakdown of particulate organic material by exposing them to microbes and their waste materials contain rich nutrients forming the food for other consumers. Thus, the macrobenthos plays a major ecological role in the mangrove ecosystem [7]. Mangroves are inhabited by a variety of macrobenthic invertebrates, such as brachyuran crabs, hermit crabs, gastropods, bivalves, barnacles, sponges, tunicates, polychaetes, and sipunculids. The mangrove invertebrates often exhibit marked zonation patterns and colonize a variety of specific micro-environments [8-10]. While some species dwell on the sediment.

\section{Materials and Methods}

Station I (Developing mangrove ecosystem) is located at $11^{\circ} 29^{\prime} \mathrm{N}$

${ }^{*}$ Corresponding author: Thilagavathi B, Faculty of Marine Science, Centre of Advanced Study in Marine Biology, Annamalai University, Parangipettai-608 502, Tamil Nadu, India, Tel: 04144-243223; Fax: 04144-243553; E-mail: thilaga_marine@yahoo.com

Received June 26, 2013; Accepted October 22, 2013; Published October 22, 2013

Citation: Thilagavathi B, Varadharajan D, Babu A, Manoharan J, Vijayalakshm S, et al. (2013) Distribution and Diversity of Macrobenthos in Different Mangrove Ecosystems of Tamil Nadu Coast, India. J Aquac Res Development 4: 199 doi:10.4172/2155-9546.1000199

Copyright: (c) 2013 Thilagavathi B, et al. This is an open-access article distributed under the terms of the Creative Commons Attribution License, which permits unrestricted use, distribution, and reproduction in any medium, provided the original author and source are credited. 
$79^{\circ} 46^{\prime} \mathrm{E}$. This is one of the best-studied estuaries in India comparable to the world conditions. It is highly productive and rich in floral and faunal resources. This located estuary remains open with the Bay of Bengal as it is a "true estuary" without complete closure of the mouth. Along the course of this estuary, in an area of 10 ha, a mangrove forest was developed by Dr. K. Kathiresan and his team, CAS in Marine Biology, Parangipettai since the year-1991 onwards. It is grown near the shore of Vellar estuary and is very good in supporting biodiversity of many species. Hence, this site was selected as a developing mangrove ecosystem

Station II (Riverine mangrove ecosystem) is located at $10^{\circ} 20^{\prime} \mathrm{N}$ $79^{\circ} 32^{\prime} \mathrm{E}$ and it is situated $400 \mathrm{~km}$ south of Chennai which lies on the southern part of Cauvery delta region along the south east coast of the Peninsular India. Avicennia marina is the dominant mangrove species in Muthupettai and accounts for nearly $95 \%$ of the vegetative cover. In Muthupettai mangrove harbours, 112 species of insects 14 species of crustaceans, 18 species of molluscs, 73 species of finfishes, 10 species of herpeto fauna and 13 species of mammals $[11,12]$.

Station III (Island mangrove ecosystem) consists of three different islands of Gulf of Mannar, namely Kurusadai, Poomarchan, and Manauli. It is located between $9^{\circ} 14^{\prime} \mathrm{N} 79^{\circ} 12^{\prime} \mathrm{E}$ and $9^{\circ} 12^{\prime} \mathrm{N} 79^{\circ} 7^{\prime} \mathrm{E}$, along the south east coast of India. These islands are well known for their mangrove species composition and have lagoon pools and open mud flats (Figure 1).

\section{Water sampling and analysis of physico-chemical parameters}

Four seasonal collections were made from January 2011 to December 2011. Samples were collected from each station (four seasons $\times$ three stations $\times$ six replicates). Rainfall data were collected from the metrological department office at CAS in Marine Biology, Annamalai University. Muthupettai for station I and Parangipettai for station II and Pamban for station III. Water samples were collected in pre-cleaned polypropylene containers, just below the water surface separately from the sampling sites. After collection, all the samples were cooled and then brought to the laboratory in an insulated thermocool box. Soon after returning to the laboratory, the water samples were filtered through a Whatman GF/C filter paper for nutrient analysis. Water temperature was measured using a mercury centigrade thermometer with $0.5^{\circ} \mathrm{C}$ accuracy. The $\mathrm{pH}$ of samples was measured

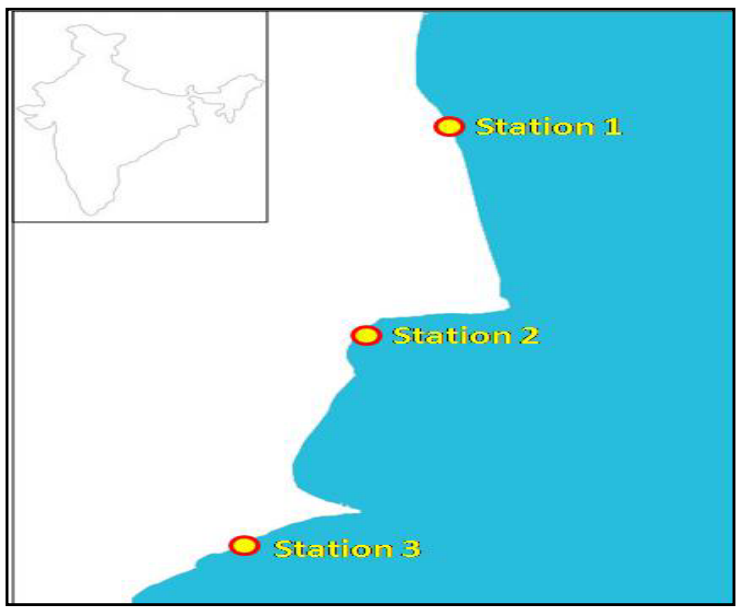

Figure 1: Location of sampled stations. by using a calibrated $\mathrm{pH}$ pen (Phep; Hanna instruments Mauritius Ltd., Portugal) with an accuracy of \pm 0.1 . The $\mathrm{pH}$ in the solution was measured using a $\mathrm{pH}$ meter, calibrated with standard buffer solution prior to use. The salinity of samples was measured by using a hand refractometer (Atago, Japan). Water samples were transferred carefully to BOD bottles. The modified Winkler's method described by Strickland JDH and Parsons TR [13] was adopted for the estimation of dissolved oxygen fixed. The nitrate, nitrite, inorganic phosphate, and reactive silicate content of water samples were analyzed by the method of Strickland JDH [13].

\section{Sediment sampling and analysis of physico-chemical parameters}

Pterson's grab $\left(0.256 \mathrm{~m}^{2}\right)$ was used to collect sediment samples. The soil temperature was measured using a standard centigrade thermometer by directly inserting in the sediment. The soil $\mathrm{pH}$ was determined by adopting the method of Jackson ML [14]. Water was added to air-dried samples in the ratio of 1:1 and stirred in a mechanical shaker for an hour, and $\mathrm{pH}$ was measured in this solution. A known amount of sediment samples was moisturized with double distilled water up to the moisture saturation of the sediment. Then double the volume of saturation level of water was added, mechanically shaken for $15 \mathrm{~min}$, and the water with salt was filtered through a Whatman No. 1 filter paper and the salinity was measured using a hand refractometer. Soil samples were brought to the laboratory in clean polythene bags, air dried, and stored for further analysis. The percentage composition of sand, silt, and clay in the sediment samples were determined by the sieving method of Krumbein WC [15].

\section{Macrofauna sampling and identification}

The samplings covered all the tidal levels and were done by using a line transect method. Pterson's grab $\left(0.256 \mathrm{~m}^{2}\right)$ was used for unit sampling. Six replicates for each station were maintained. Soon after retrieval, samples were gently sieved through a $0.5-\mathrm{mm}$ sieve. The organisms retained by the sieve were preserved in $5 \%$ formalin and brought to the laboratory for further identification. The sorted organisms were first segregated into different groups and then identified to specific, genetic or other higher levels to the greatest extent possible with the help of standard taxonomic references viz. Polychaeta $[16,17]$ and Mollusca [18]. The organisms were counted under a stereoscopic microscope, and abundance was expressed as individuals per square meter. In the present study, the qualitative and quantitative assessments of benthic macrofauna were noted only to polychaetes, bivalves, and gastropods (molluscs), and isopods and amphipods (crustaceans).

\section{Data analysis}

Macrofauna taxa collected from the beds were identified and listed. Pearson correlation coefficient was employed for the better understanding of relationship between the concentration of various nutrients, sediment composition, and $\mathrm{pH}$ by using statistical package (SPSS-11.5). Their settlement was analyzed using several indices: univariate measures such as Margalef 's species richness (d), ShannonWiener diversity $\left(\mathrm{H}^{\prime} \log \right)$ and Pielou's evenness $\left(\mathrm{J}^{\prime}\right)$, graphical tools like $\mathrm{k}$-dominance curve and multivariate tool such as Bray-Curtis similarity after suitable transformation of sample abundance data, classification (hierarchical agglomerative clustering using group-average linking), and ordination (multidimensional scaling, MDS) were used for treating the data and were calculated using of computer software of PRIMER (Ploymouth Routines In Multivariate Ecological Research ver. 6). 

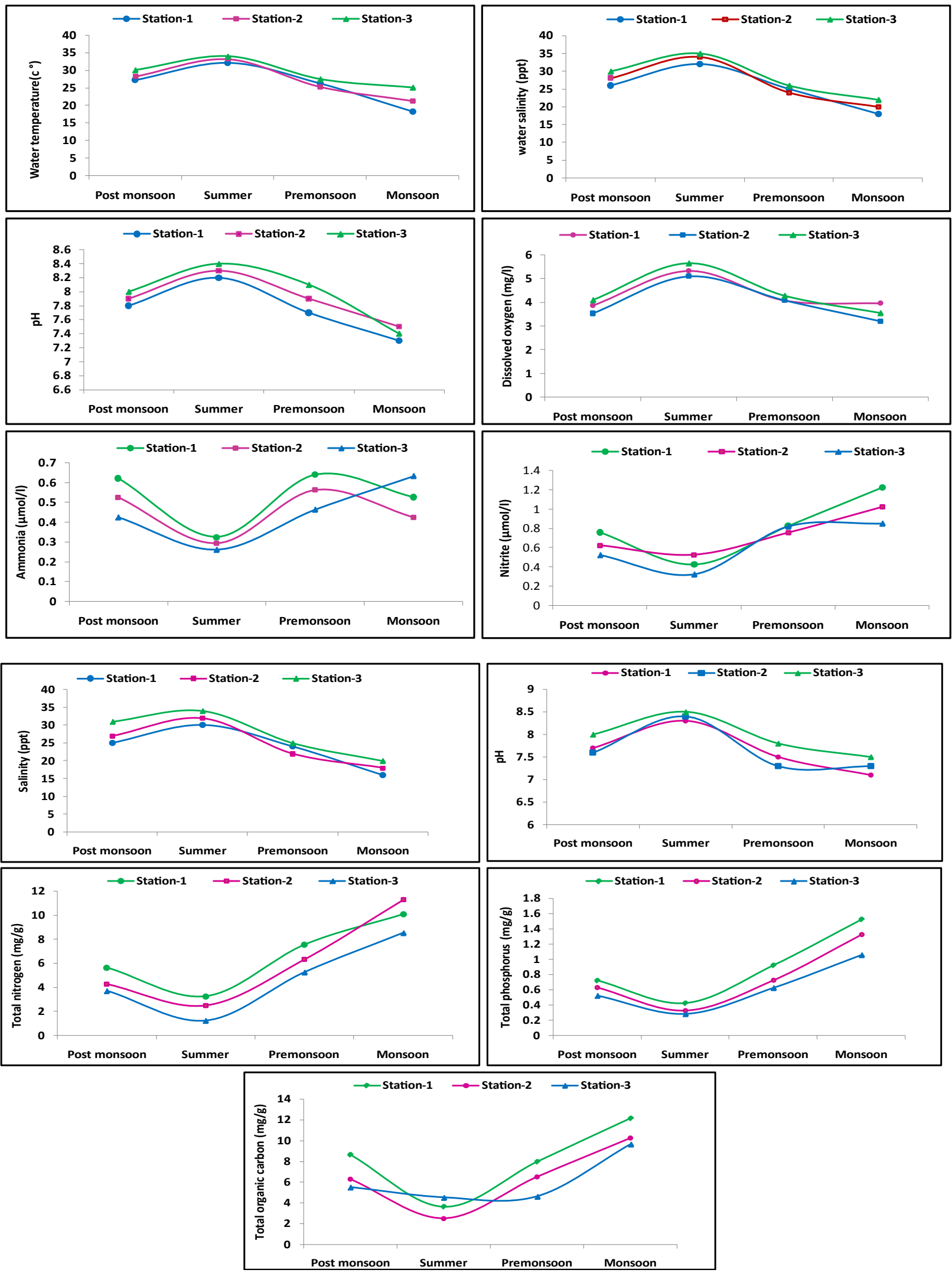

Figure 2: Seasonal variations of physico-chemical parameters in water and sediment samples. 
Citation: Thilagavathi B, Varadharajan D, Babu A, Manoharan J, Vijayalakshmi S, et al. (2013) Distribution and Diversity of Macrobenthos in Different Mangrove Ecosystems of Tamil Nadu Coast, India. J Aquac Res Development 4: 199 doi:10.4172/2155-9546.1000199

\section{Results}

\section{Environmental parameters}

The physical parameters of water and sediment were similar in all stations (I, II \& III) throughout the experimental period, indicating the well-mixed nature of this ecosystem. The value of rainfall ranged from 17.37 to $4,400 \mathrm{~mm}$ in all stations. Temperature, salinity, and $\mathrm{pH}$ of both water and sediments ranged from $18.2^{\circ} \mathrm{C}$ to $30.1^{\circ} \mathrm{C}$ and $20.1^{\circ} \mathrm{C}$ to $35.1^{\circ} \mathrm{C}, 18$ to $35 \mathrm{ppt}$ and 16 to $34 \mathrm{ppt}$, and 7.3 to 8.4 and 7.1 to 8.5 respectively. The values of dissolved oxygen ranged from 3.22 to 5.65 $\mathrm{mg} / \mathrm{l}$. Nutrients in water such as ammonia, nitrite, nitrate, total nitrogen inorganic phosphate, total phosphorus and reactive silicate ranged from 0.263 to $0.654 \mu \mathrm{mol} / \mathrm{l} 0.326$ to $1.226 \mu \mathrm{mol} / \mathrm{l}, 1.263$ and $5.563 \mu \mathrm{mol} / \mathrm{l}$, 3.25 to $15.637 \mu \mathrm{mol} / \mathrm{l}, 0.128$ to $0.622 \mu \mathrm{mol} / \mathrm{l}, 0.285$ to $1.526 \mu \mathrm{mol} / \mathrm{l}$ and 6.248 to $24.526 \mu \mathrm{mol} / \mathrm{l}$ respectively. In sediments nutrients such as nitrogen, phosphorus, and total organic carbon were recorded, and these are varied from 1.263 to $11.258 \mu \mathrm{g} / \mathrm{g}, 2.517$ to $12.132 \mu \mathrm{g} / \mathrm{g}$, and 2.517 to $12.132 \mu \mathrm{g} / \mathrm{g}$ respectively (Figure 2 ). The sediment texture in terms of sand, clay, and silt (\%) were 1.18-69.87, 2.64-26.82, and 8.9495.48 in all the three stations (Figure 2).

\section{Species composition of macrofauna}

A total of 292 macrobenthic faunal species represented by six diverse groups were encountered, of which polychaetes, gastropods, bivalves, amphipods, isopods and cumacea were the most important groups. Polychaetes are dominated in the macrobenthic fauna (188 species) and contributed numerically up to $64.38 \%$ of the population. Bivalves consist of 12 species and contribute to $4.11 \%$ of the total fauna production. Gastropods consist of 17 species and contribute to $5.82 \%$ of the total fauna production. Amphipods consist of 55 species and contribute to $18.83 \%$ of the total fauna production. Isopods consist of 16 species and contribute to $5.47 \%$ of the total fauna production. Also, cumaceans include 4 species and contribute to $1.37 \%$ of the total faunal production (Figure 3).

The 252 species ( 151 polychaetes, 12 bivalves, 16 gastropods, 53 amphipods, 16 isopods and 4 cumaceans) were recorded in station I, and the percentage composition was calculated and shown in Figure 4. The 156 species (102 polychaetes, 10 bivalves, 11 gastropods, 24 amphipods, 6 isopods and 3 cumaceans) and 163 species (105 polychaetes, 10 bivalves, 16 gastropods, 21 amphipods, 9 isopods and 2 cumaceans) were recorded in stations II and III respectively. The percentage composition was calculated for both stations and presented in Figures 5 and 6 . The species belonging to all groups are presented in Table 1. Bivalves and crustaceans were dominant in faunal biomass. The highest number of species was recorded in station II than in others. The benthic macrofaunal density (ind $/ \mathrm{m}^{2}$ ) was calculated and ranged from 156 to 217 , from 84 to 171 , and from 99 to 171 in stations I, II, and III, respectively. The highest benthic macrofaunal density was recorded in the early summer season at station I.

Classification analyses (using Bray-Curtis similarity) followed by an ordination through MDS on benthos abundance data (No/0.256 $\mathrm{m}^{2}$ ) independently for fauna (293 species) were undertaken. The 12 investigation stations (four seasons $\times$ three stations) have been divided into three groups: S1Pm, S2Sm, S3PrM, S4Mn; S5Pm, S6Sm, S7PrM, S8Mn; and S9Pm, S10Sm, S11PrM, S12Mn corresponding to Muthupettai (station I), Parangipettai (station II), and the Gulf of Mannar (station III). Figures 7 and 8 display results of MDS ordination and hierarchical clustering, respectively, on species abundance data representing the three stations during four seasons (post-monsoon, summer, pre-monsoon, and monsoon). Cluster analysis showed that the macrofauna communities at each of the mangrove communities were relatively most similar (Figure 9). The $2 \mathrm{D}$ stress value (0.11) indicated that the results are credible. The station I and III communities, which are very similar in the result of the cluster analysis, were clearly separated.

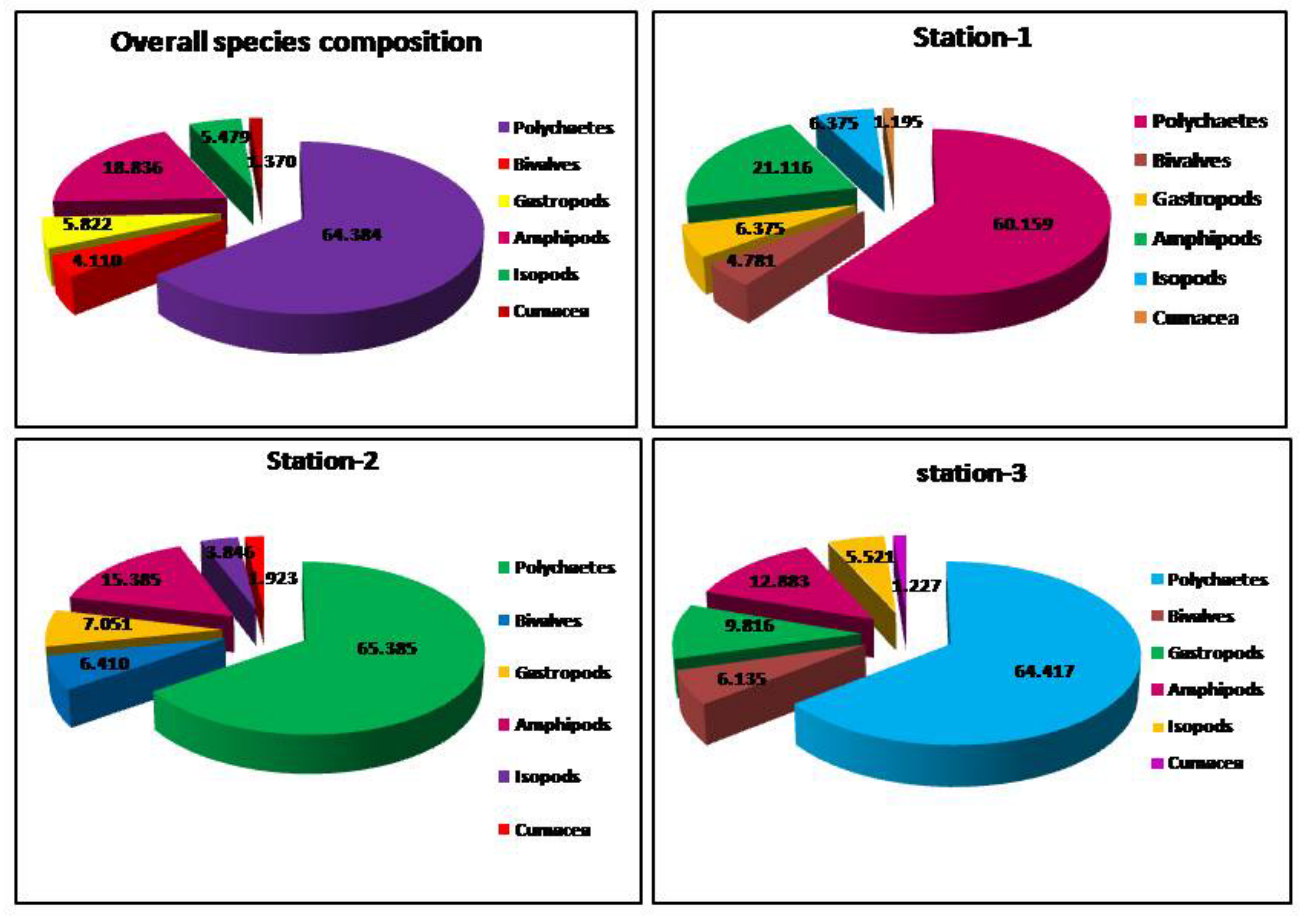

Figure 3: Percentage composition of macrobenthos in different stations. 
Citation: Thilagavathi B, Varadharajan D, Babu A, Manoharan J, Vijayalakshmi S, et al. (2013) Distribution and Diversity of Macrobenthos in Different Mangrove Ecosystems of Tamil Nadu Coast, India. J Aquac Res Development 4: 199 doi:10.4172/2155-9546.1000199

Page 5 of 12

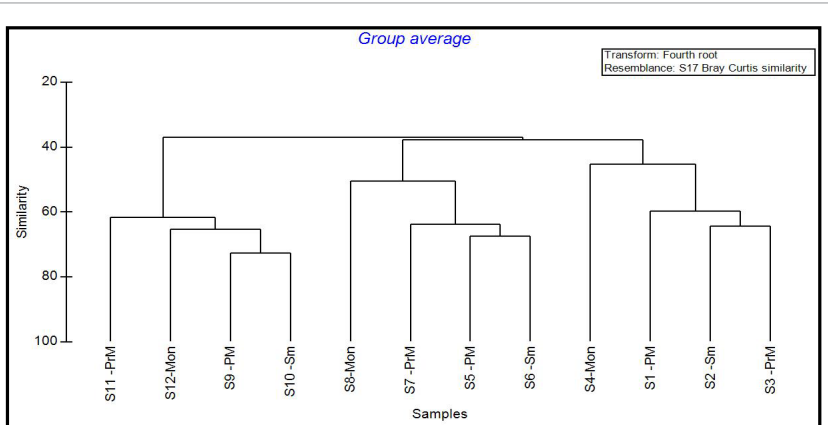

Figure 4: Dendrogram showing the similarity between stations and all the seasons.

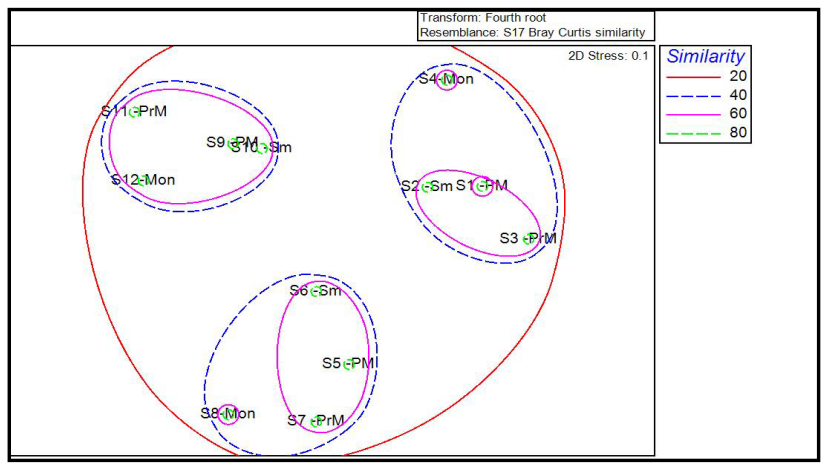

Figure 5: MDS Plot for all the seasons.

In comparison, among the three sampling stations, station II communities were the shortest, implying that the structure of macrofauna communities at this community was the most similar (Figure 9).

From the resulting dendrogram (Figure 4), it is possible to classify the results according to stations and also for seasons. Station III is separated from the others. In the MDS plot (Figure 4), it is found that all season samples are separated conforming to the dendrogram. The benthic faunal density (N) (Figure 5A) varied from 84 (station II, monsoon) to 217 (station I, summer). The Shannon-Wiener index (H) (Figure $5 \mathrm{~B}$ ) ranges between 4.311(station II, monsoon) and 5.167 (station I, summer). The evenness component $\left(\mathrm{J}^{\prime}\right)$ (Figure 5D) varied from 0.998 (station III, summer) to 0.999 (station I, monsoon). It is low during the post-monsoon and summer season and gradually increases during the monsoon seasons. The richness component (D) (Figure 5C) ranged between 16.69 (station II, monsoon) to 32.70 (station I, summer).

Multiple k-dominance plots facilitate the discrimination of benthos according to species-relative contribution to standard stock. The k-dominance curves obtained for different stations show higher diversity except S8Sm. The stations S2Sm and S1PM show maximum diversity as the curves for all three stations are lying lower than others whereas the curve of the $\mathrm{S} 12 \mathrm{M}$ is lying in the top and has a stiff elevation indicating the lowest diversity (Figure 6).

The k-dominance plot is plotted according to station (Figure 7); it shows the plot for pooled data, i.e., it shows a perfect $S$ curve indicating the high diversity of macrofauna in station I without disturbance, when the curves were drawn separately for the three stations among the seasons. The k-dominance plot is also plotted for all the seasons, and the curve drawn inputting all the stations and all the seasons are

\begin{tabular}{|c|c|c|c|c|}
\hline S. No & Species & St-1 & St-2 & St-3 \\
\hline I & Polychaetes & & & \\
\hline 1 & Abarenicola gilchristi & * & * & * \\
\hline 2 & Gattyana deludens & * & - & - \\
\hline 3 & Nephtys bucera & * & - & * \\
\hline 4 & Nereis abbreviata & * & * & * \\
\hline 5 & Nereis diversicolor & * & * & * \\
\hline 6 & Nereis jacksoni & * & * & * \\
\hline 7 & Onuphis eremite & * & * & * \\
\hline 8 & Scolelepis squamata & * & - & - \\
\hline 9 & Abarenicola gilchristi & * & * & * \\
\hline 10 & Amphicteis gunneri & - & - & * \\
\hline 11 & Amphinome rostrata & * & - & - \\
\hline 12 & Ancistrosyllis constricta & * & * & * \\
\hline 13 & Ancistrosyllis groenlandica & * & * & - \\
\hline 14 & Ancistrosyllis parva & * & * & * \\
\hline 15 & Arabella iricolor & * & * & - \\
\hline 16 & Arenicola loveni & * & - & - \\
\hline 17 & Armandia lanceolata & - & - & * \\
\hline 18 & Armandia longicaudata & * & * & * \\
\hline 19 & Axiothella obockenisis & * & * & - \\
\hline 20 & Bhawania goodei & * & - & - \\
\hline 21 & Branchiocapitella singularis & * & - & - \\
\hline 22 & Capitella capitata & - & * & * \\
\hline 23 & Ceratonereis costae & * & * & * \\
\hline 24 & Ceratonereis keiskama & * & - & - \\
\hline 25 & Ceratonereis mirabilis & * & * & - \\
\hline 26 & Chaetozone setosa & * & - & - \\
\hline 27 & Chloeia flava & - & * & * \\
\hline 28 & Chloeia parva & * & - & * \\
\hline 29 & Chone collaris & * & * & * \\
\hline 30 & Chone filicaudata & * & * & * \\
\hline 31 & Cirratulus chrysoderma & * & * & * \\
\hline 32 & Cirratulus concinnus & * & * & * \\
\hline 33 & Cirratulus gilchristi & - & * & * \\
\hline 34 & Cirriformia tentaculata & * & * & * \\
\hline 35 & Cossura delta & * & * & * \\
\hline 36 & Dasychone cingulata & * & - & - \\
\hline 37 & Dendronereis aestuarina & * & - & - \\
\hline 38 & Dendronereis arborifera & * & - & * \\
\hline 39 & Diopatra cuprea & * & * & * \\
\hline 40 & Diopatra neapolitana & * & - & - \\
\hline 41 & Disoma orissae & * & - & * \\
\hline 42 & Dorvillea incertus & * & * & * \\
\hline 43 & Dorvillea neglecta & * & - & * \\
\hline 44 & Enice pennata & - & * & * \\
\hline 45 & Enice tentaculata & * & * & * \\
\hline 46 & Epidiopatra hupferiana & - & * & * \\
\hline 47 & Eteone ornata & - & - & - \\
\hline 48 & Eteone ornata & * & - & * \\
\hline 49 & Eteone siphodonta & - & * & - \\
\hline 50 & Euchone rosea & * & - & - \\
\hline 51 & Euclymene annandalei & * & * & - \\
\hline 52 & Eulalia macroceros & * & - & * \\
\hline 53 & Eulalia sanguinea & - & * & - \\
\hline 54 & Eunice australis & * & - & - \\
\hline 55 & Eunice indica & - & * & * \\
\hline 56 & Eunice tubifex & * & - & * \\
\hline 57 & Euphrosine capensis & - & * & - \\
\hline 58 & Eurythoe complanata & * & * & - \\
\hline 59 & Exogone clavator & * & * & * \\
\hline
\end{tabular}


Citation: Thilagavathi B, Varadharajan D, Babu A, Manoharan J, Vijayalakshmi S, et al. (2013) Distribution and Diversity of Macrobenthos in Different Mangrove Ecosystems of Tamil Nadu Coast, India. J Aquac Res Development 4: 199 doi:10.4172/2155-9546.1000199

Page 6 of 12

\begin{tabular}{|c|c|c|c|c|}
\hline 60 & Exogone verugera & - & * & * \\
\hline 61 & Fabriciola mossambica & * & * & * \\
\hline 62 & Gattyana deludens & - & * & - \\
\hline 63 & Glycera africana & * & * & - \\
\hline 64 & Glycera alba & - & * & * \\
\hline 65 & Glycera longipinnis & * & - & - \\
\hline 66 & Glycera onicornis & * & * & * \\
\hline 67 & Glycinde capensis & * & * & * \\
\hline 68 & Glycinde oligodon & * & * & - \\
\hline 69 & Goniada emerita & - & - & * \\
\hline 70 & Goniada goniada & * & * & * \\
\hline 71 & Goniadella gracilis & * & - & - \\
\hline 72 & Goniadopsis maskallensis & - & - & * \\
\hline 73 & Harmothoe africana & * & - & * \\
\hline 74 & Hesione interexta & - & * & - \\
\hline 75 & Heteromastus similis & * & - & - \\
\hline 76 & Hololepidella maculata & - & - & * \\
\hline 77 & Hydroides albiceps & * & * & - \\
\hline 78 & Hydroides heteroceros & - & * & - \\
\hline 79 & Hydroides homoceros & * & - & * \\
\hline 80 & Isolda pulchella & - & * & * \\
\hline 81 & Lanice socialis & * & - & - \\
\hline 82 & Laonice cirrata & * & * & - \\
\hline 83 & Laonome indica & - & - & * \\
\hline 84 & Leanira hystricis & * & - & - \\
\hline 85 & Leocrates claparedii & - & * & * \\
\hline 86 & Lepidonotus tenuisetosus & * & * & - \\
\hline 87 & Loimia medusa & - & - & * \\
\hline 88 & Lopadorhynchus henseni & * & - & - \\
\hline 89 & Lopadorhynchus nationalis & * & - & * \\
\hline 90 & Lumbriconereis impatiens & - & * & - \\
\hline 91 & Lumbriconereis latreilli & * & - & * \\
\hline 92 & Lumbriconereis polydesma & * & * & - \\
\hline 93 & Lumbriconereis heteropoda & - & - & * \\
\hline 94 & Lumbriconereis latreilli & * & - & * \\
\hline 95 & Lumbriconereis pseudobifilaris & - & * & * \\
\hline 96 & Lumbriconereis simplex & - & - & * \\
\hline 97 & Lumbrinereis brevicirra & * & * & * \\
\hline 98 & Lumbrinereis magalhaensis & * & * & * \\
\hline 99 & Magelona cincta & * & - & * \\
\hline 100 & Magelona papillicornis & * & - & * \\
\hline 101 & Malacoceros indicus & * & - & - \\
\hline 102 & Maldane sarsi & * & - & * \\
\hline 103 & Marphysa gravelyi & * & * & * \\
\hline 104 & Mercierella enigmatica & * & * & - \\
\hline 105 & $\begin{array}{l}\text { Mesochaetopterus mesochaetop- } \\
\text { terus }\end{array}$ & * & - & - \\
\hline 106 & Nephtys capensis & * & * & * \\
\hline 107 & Nephtys polybranchia & * & * & * \\
\hline 108 & Nephtys sphaerocirrata & * & * & * \\
\hline 109 & Nereis granulata & * & * & * \\
\hline 110 & Nereis vireins & * & * & * \\
\hline 111 & Notocirrus brevicirrus & * & - & * \\
\hline 112 & Notomastus aberans & * & * & * \\
\hline 113 & Notomastus fauveli & * & * & * \\
\hline 114 & Notomastus giganteus & * & - & * \\
\hline 115 & Onuphis eremita & * & - & * \\
\hline 116 & Onuphis geophiliformis & * & * & * \\
\hline 117 & Onuphis holobranchiata & * & * & - \\
\hline 118 & Ophelia africana & * & - & * \\
\hline 119 & Ophelia capensis & * & * & - \\
\hline
\end{tabular}

\begin{tabular}{|c|c|c|c|c|}
\hline 120 & Orbinia angrapequensis & * & * & - \\
\hline 121 & Oriopsis eimeri & - & * & * \\
\hline 122 & Oriopsis neglecta & * & - & - \\
\hline 123 & Owenia fusiformis & * & * & * \\
\hline 124 & Paralacydonia weberi & * & - & - \\
\hline 125 & Pectinaria crassa & * & - & - \\
\hline 126 & Pectinaria crassa & * & - & - \\
\hline 127 & Pelagobia longicirrata & * & - & - \\
\hline 128 & Pelagobia longicirrata & * & * & - \\
\hline 129 & Perinereis cultrifera & * & * & * \\
\hline 130 & Phyllodoce longipes & - & * & * \\
\hline 131 & Phyllodoce madeirensis & * & - & - \\
\hline 132 & Phyllodoce malmgreni & * & - & - \\
\hline 133 & Phyllodoce tenuiss & - & * & * \\
\hline 134 & Phyllodoce tubicola & * & - & * \\
\hline 135 & Pisione africana & * & * & - \\
\hline 136 & Pisionidens indica & * & - & * \\
\hline 137 & Pista quadrilobata & * & * & - \\
\hline 138 & Pista typha & * & - & * \\
\hline 139 & Platynereis calodonta & * & - & - \\
\hline 140 & Poecilochaetus serpens & * & * & - \\
\hline 141 & Polycirrus plumosus & - & * & * \\
\hline 142 & Polycirrus tribullata & * & - & - \\
\hline 143 & Polydora ciliata & * & * & * \\
\hline 144 & Polydora hophura & * & * & * \\
\hline 145 & Polydra ciliata & * & * & * \\
\hline 146 & Pomatoceros triqueter & * & - & * \\
\hline 147 & Pomatoleios kraussii & * & * & - \\
\hline 148 & Prionospio cirrifera & * & - & * \\
\hline 149 & Prionospio cirrobranchiata & * & * & * \\
\hline 150 & Prionospio malmgreni & * & * & - \\
\hline 151 & Prionospio pinnata & * & * & * \\
\hline 152 & Prionospio polybranchiata & * & - & * \\
\hline 153 & Prionospio saldanha & * & * & * \\
\hline 154 & Protodorvillea egena & - & - & * \\
\hline 155 & Pseudonereis variegata & * & * & - \\
\hline 156 & Pseudopolydora kempi & - & * & - \\
\hline 157 & Rhodine gracilior & * & - & - \\
\hline 158 & Sabellaria cementarium & * & * & - \\
\hline 159 & Sabellaria intoshi & * & - & - \\
\hline 160 & Sabellaria spimnulosa & - & - & * \\
\hline 161 & Scolelepis squamata & * & * & * \\
\hline 162 & Scoloplella capensis & * & * & - \\
\hline 163 & Scoloplella capensis & * & - & * \\
\hline 164 & Scoloplos marsupialis & * & - & * \\
\hline 165 & Serpula vermicularis & * & - & * \\
\hline 166 & Sigalion squamatum & - & - & - \\
\hline 167 & Sphaerosyllis erinaceu & * & - & - \\
\hline 168 & Spio filicornis & * & - & * \\
\hline 169 & Spiochaetopterus costarum & * & * & * \\
\hline 170 & Spirobrachus teraceros & - & - & - \\
\hline 171 & Spirorbis foraminosus & * & * & - \\
\hline 172 & Sternaspis scutata & * & - & * \\
\hline 173 & Sthenelais boa & * & * & - \\
\hline 174 & Sthenelais japonica & - & - & \\
\hline 175 & Stylarioides stylarioides & * & - & - \\
\hline 176 & Syllidia armata & * & * & - \\
\hline 177 & Syllis benguellana & * & - & * \\
\hline 178 & Syllis gracilis & * & * & * \\
\hline 179 & Syllis longocirrata & * & * & * \\
\hline 180 & Syllis trifalcata & * & * & - \\
\hline
\end{tabular}


Citation: Thilagavathi B, Varadharajan D, Babu A, Manoharan J, Vijayalakshmi S, et al. (2013) Distribution and Diversity of Macrobenthos in Different Mangrove Ecosystems of Tamil Nadu Coast, India. J Aquac Res Development 4: 199 doi:10.4172/2155-9546.1000199

Page 7 of 12

\begin{tabular}{|c|c|c|c|c|}
\hline 181 & Terebella pterochaeta & * & - & - \\
\hline 182 & Terebellides stroemi & * & * & - \\
\hline 183 & Thelepus setosus & * & - & - \\
\hline 184 & Tlonereis fauveli & * & - & - \\
\hline 185 & Tomopteris helgolandica & * & * & - \\
\hline 186 & Travisiopsis lobifera & * & * & - \\
\hline 187 & Typhloscolex muelleri & * & - & - \\
\hline 188 & Vanadis formosa & * & - & - \\
\hline II & Bivalves & & & \\
\hline 189 & Anadara granosa & * & * & * \\
\hline 190 & Anadara rhombea & * & * & * \\
\hline 191 & Cardium setosum & * & * & * \\
\hline 192 & Donax scortum & * & * & * \\
\hline 193 & Donax cuneatus & * & * & * \\
\hline 194 & Donax spinosus & * & * & * \\
\hline 195 & Meretrix meretrix & * & - & * \\
\hline 196 & Modiolus metcalfei & * & * & * \\
\hline 197 & Perna viridis & * & - & * \\
\hline 198 & Pincdata fucata & * & * & * \\
\hline 199 & Placenta placenta & * & * & - \\
\hline 200 & Paphia malabarica & * & * & - \\
\hline III & Gastropods & & & \\
\hline 201 & Bullia vitata & * & * & * \\
\hline 202 & Cerithedia cingulata & * & * & * \\
\hline 203 & Cerithedia obtusa & * & * & * \\
\hline 204 & Epitonium scalare & * & * & - \\
\hline 205 & Littorina scabra & * & * & * \\
\hline 206 & Nassarius variegatus & * & * & * \\
\hline 207 & Natica tigerina & * & - & * \\
\hline 208 & Oliva nebulosa & * & - & * \\
\hline 209 & Turritella attenuata & * & * & * \\
\hline 210 & Turritella albenuata & * & - & * \\
\hline 211 & Turritella acqutangula & * & - & * \\
\hline 212 & Umbonium vestiarium & * & * & * \\
\hline 213 & Telescopium telescopium & * & * & * \\
\hline 214 & Murex tribulex & * & * & * \\
\hline 215 & Nassa jacksoniana & * & - & * \\
\hline 216 & Nassarius scabra & * & * & * \\
\hline 217 & Oliva nebulosa & - & - & * \\
\hline IV & Amphipods & & & \\
\hline 218 & Ampelisca scabripes & * & * & - \\
\hline 219 & Ampithoe rubricata & * & * & - \\
\hline 220 & Ampithoerubricata & * & * & * \\
\hline 221 & Ampithogammaroides & * & * & * \\
\hline 222 & Atylus falcatus & - & * & - \\
\hline 223 & Caprella mendax & * & * & * \\
\hline 224 & Cerapus crassicornis & * & - & * \\
\hline 225 & Cheiriphotis megacheles & * & - & - \\
\hline 226 & Corophium triaenonyx & * & * & - \\
\hline 227 & Cymadusa pathyi & * & - & - \\
\hline 228 & Erichthonius brasiliensis & * & - & - \\
\hline 229 & Eriopisa abhilashi & * & - & - \\
\hline 230 & Eriopisa chilkensis & * & - & - \\
\hline 231 & Gammaropsis esturinus & * & * & * \\
\hline 232 & Gammaropsis maculata & * & * & * \\
\hline 233 & Gammarus duebeni & * & * & * \\
\hline 234 & Gammarus zaddachi & * & * & * \\
\hline 235 & Gitanopsis bispinosa & * & * & - \\
\hline 236 & Gitanopsis gouriae & * & - & - \\
\hline 237 & Grandidierella bispinosa & * & * & * \\
\hline 238 & Grandidierella bonnieroides & * & - & - \\
\hline
\end{tabular}

\begin{tabular}{|c|c|c|c|c|}
\hline 239 & Grandidierella gilesi & * & - & * \\
\hline 240 & Grandidierella gravipes & * & * & - \\
\hline 241 & Grandidierella macronyx & * & * & * \\
\hline 242 & Grandidierella megnae & * & - & - \\
\hline 243 & Harnellia incerta & * & - & * \\
\hline 244 & Harpinia antennaria & * & - & - \\
\hline 245 & Harpinia laevis & * & - & - \\
\hline 246 & Harpinia Pectinata & * & - & - \\
\hline 247 & Hornellia incerta & * & - & - \\
\hline 248 & Hyale honoluluensis & * & - & - \\
\hline 249 & Idunella chilkensis & * & - & - \\
\hline 250 & Ingolfiella putealis & * & - & - \\
\hline 251 & Jassa falcata & * & - & - \\
\hline 252 & Jassa marmorata & - & - & - \\
\hline 253 & Maera othonides & * & * & * \\
\hline 254 & Metaphoxus fultoni & * & * & - \\
\hline 255 & Metaphoxus pectinatus & * & * & - \\
\hline 256 & Microporotopus maculatus & * & - & - \\
\hline 257 & Microprotopus cumbreansis & * & - & - \\
\hline 258 & Natarajphotis manieni & * & * & * \\
\hline 259 & Orchestia platenis & * & * & - \\
\hline 260 & Paracalliope indica & * & - & - \\
\hline 261 & Parhyale hawaiensis & * & - & - \\
\hline 262 & Parorchestia morini & * & - & - \\
\hline 263 & Parorchestia notabilis & * & - & - \\
\hline 264 & Photis digitata & * & - & * \\
\hline 265 & Phoxocephalus holbolli & * & * & * \\
\hline 266 & Podoecerus brasiliensis & * & - & * \\
\hline 267 & Pontharpinia rostrata & * & * & * \\
\hline 268 & Quadrivisio bengalensis & * & - & - \\
\hline 269 & Talorchestia martensii & * & - & - \\
\hline 270 & Urothoe pulchella & * & * & * \\
\hline 271 & Urothoe serrudactyla & * & * & * \\
\hline 272 & Urothoe viswanathi & * & - & * \\
\hline V & ISOPODS & & & \\
\hline 273 & Angeliera phreaticola & * & * & * \\
\hline 274 & Basserolis kimblae & * & - & - \\
\hline 275 & Calabozoa pellucida & * & - & - \\
\hline 276 & Eisothistos antarcticus & * & * & * \\
\hline 277 & Haploniscus laticephalus & * & - & - \\
\hline 278 & Jaeropsis beuroisi & * & - & - \\
\hline 279 & Janaira gracilis & * & - & - \\
\hline 280 & Microjaera anisopoda & * & * & * \\
\hline 281 & Paragnathia formica & * & * & * \\
\hline 282 & Sphaeroma serratum & * & - & * \\
\hline 283 & Munna boecki & * & - & - \\
\hline 284 & Plurocope dasyura & * & - & * \\
\hline 285 & Eurydice pulchra & * & - & * \\
\hline 286 & Microjaera anisopoda & * & * & - \\
\hline 287 & Cymodoce truncata & * & - & * \\
\hline 288 & Anthura gracilis & * & * & * \\
\hline VI & Cumacea & & & \\
\hline 289 & Campylaspis minor & * & * & - \\
\hline 290 & Nannastacus inflatus & * & - & * \\
\hline 291 & Gynodiastylis lata & * & * & * \\
\hline \multirow[t]{2}{*}{292} & Picrocuma poecilata & * & * & - \\
\hline & Total & 251 & 156 & 163 \\
\hline
\end{tabular}

Table 1: Species Recorded During the Study Period of January 2011 to December 2011. 

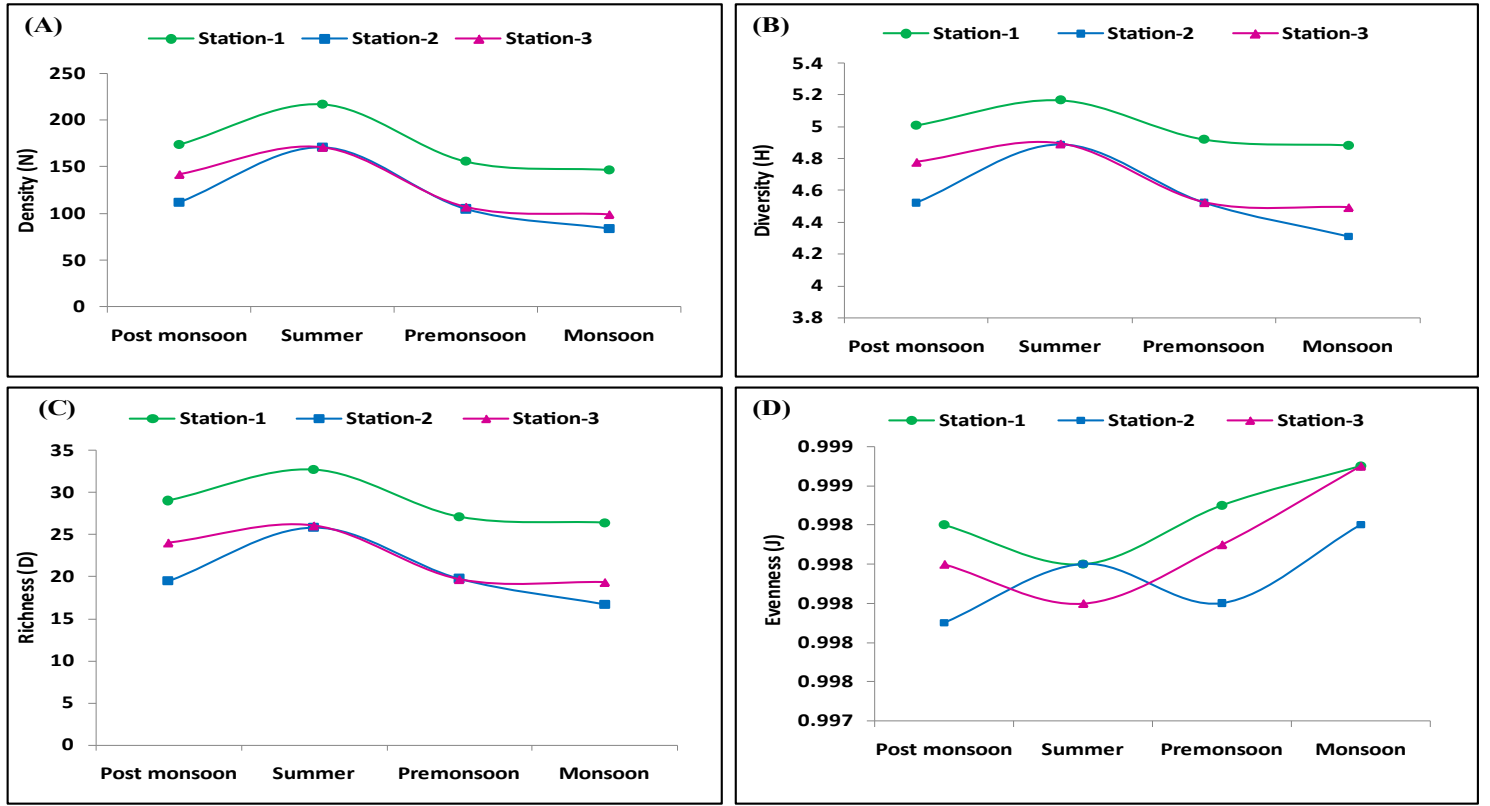

Figure 6: Univariate Measures for Macro-Benthic Macrofauna of Study Area (season-wise). A. Species density (N), B. Shannon Wiener diversity (H), C. Margalf richness (D), D. Pielou's evenness (J).

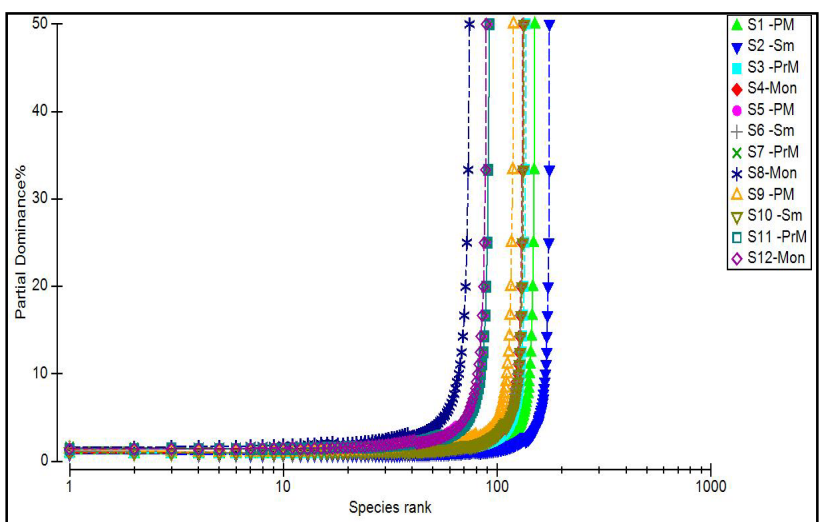

Figure 7: K-Dominance curves for all stations and seasons.

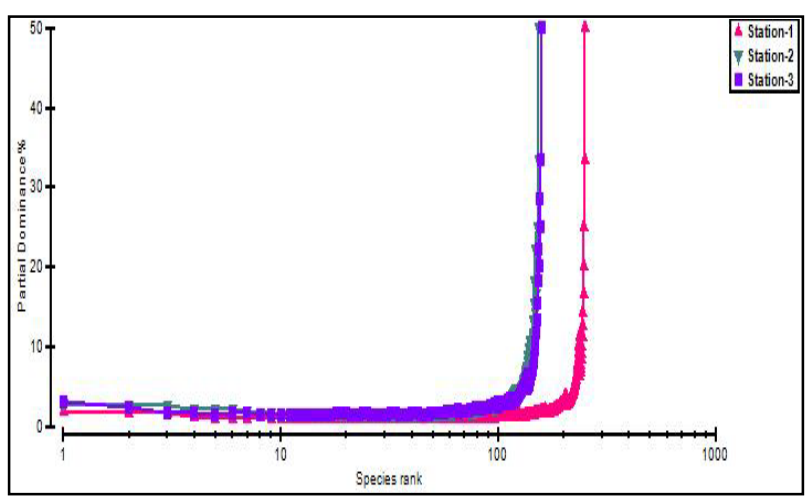

Figure 8: K-Dominance curves for all stations.

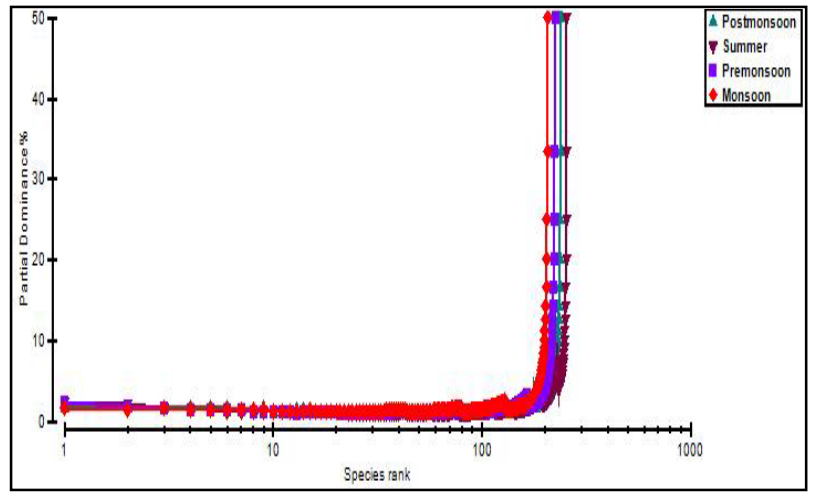

Figure 9: K-Dominance curves drawn for all the four seasons.

shown (Figure 8). The curve representing during the monsoon season lies at the top indicating lower diversity and curve represented during the summer season at the bottom indicating a higher diversity. Other two seasons fall in between these two seasons; the $\mathrm{S}$ shape of the graph is clear evidence that there is no disturbance to these resources.

\section{Discussion}

One of the main goals of benthic ecology has been to understand the mechanisms regulating relationships between physico-chemical parameter and organisms [19-22]. The present study shows that the macrofaunal communities of three mangrove ecosystems exhibit distinct variations. It is a characterized by temporal and spatial changes in its population and distribution pattern seems to be fully governed by the physico-chemical and hydrobiological characteristics of the environment. Intertidal fauna at the study area have to cope with harsh environmental conditions marked by high salinity, increased 
evaporation, wide seasonal temperature fluctuations, and different degrees of tidal amplitudes. These unique physico-chemical factors exert a strong influence on faunal assemblages, which are withstanding the situation. Owing to the heterogeneous nature of estuarine water, the relatively stationary benthic animals on the bottom have to endure a wide range of environmental changes when the circulation carries different kinds of water over the site or borrow [23]. Mangroves also possess some positive advantages of benthic animals, compared to the open coast. Estuaries are relatively sheltered against wind waves and ocean swell; most estuaries are also rich in food provided by river input, input from mangroves, and high primary production [24,25].

This study has shown that there is difference in macrobenthic fauna at different mangrove types like developing, riverine, and island mangroves. The structure of benthic macrofauna communities is characterized by a low abundance and a very low diversity. Richer communities have been found in station I. The macrobenthic faunal density ranges from 84 to $217 \mathrm{ind} / \mathrm{m}^{2}$ in all the stations. This density is higher than the macrobenthic faunal densities as reported by Parulekar $\mathrm{AH}$ [26] for Zuari estuary (50 to $1,437 \mathrm{ind} / \mathrm{m}^{2}$ ), by Parulekar $\mathrm{AH}$ and Ansari ZA [27] for Andaman seas ( 80 to $998 \mathrm{ind} / \mathrm{m}^{2}$ ), and comparable with that of Harikantra SN et al. [28] who record the density range of macrobenthos from 50 to $3,715 \mathrm{ind} / \mathrm{m}^{2}$ in the shelf region along the west coast of India. However, this value is lower than the reported density of 1,253 to $5,723 \mathrm{ind} / \mathrm{m}^{2}$ in northwestern Arabian Sea shelf by Parulekar $\mathrm{AH}$ [26] in northern sea. The difference in the benthic macrofaunal densities of different aquatic systems could be attributed mainly to variations in salinity, substratum and sediment organic carbon level, currents, and predation.

The species composition of benthic macrofauna in the present observation shows the dominance of polychaete followed by molluscs and crustaceans. Similar preponderance of polychaetes has been observed earlier by Sankar G [29] in Muthupet lagoon, Sunil Kumar [30] in Cochin backwaters, Prabha Devi L [31] in Coleroon estuary, and Ansari ZA et al. [32] in Mandovi estuary. Athalye RP and Gokhale KS [33] reported the dominance of polychaetes followed gastropods, bivalves, and hermit crabs in the Thane creek, Mumbai. The benthic population density shows seasonal variation in such a way that the maximum is recorded in summer and the minimum during monsoon at all the stations. The dominance of polychaetes might be due to firm substrate provided by roots and dense canopy of the mangroves which also provide protection against desiccation [34]. They have more opportunistic bearing potential ability to colonize in stressed environments [4]. The aforementioned adaptable nature of polychaetes may be a plausible reason for their dominance in the species composition and their abundance in the present investigation. In the present study, mollusks form the second dominant group followed by polychaetes. The dominance of gastropods and bivalves are also observed by Kathiresan K et al. [35] in Vellar estuary on the southeast coast of India. They report that high tolerance to different environmental situation and various estuarine conditions reveal its higher abundance. In the present study, crustaceans form the third group after polychaetes and molluscs. The present observation shows numerical dominance in the decreasing order as polychaetes, mollusks (bivalves and gastropods), and crustaceans, as observed earlier by Mohammed SZ and Kumar RS $[36,37]$ in other mangrove environs of India. Irrespective of mangrove types, the mangroves show the same order of polychaetes, molluscs, and crustaceans. From this, it is evident that polychaetes form the dominant group of macrobenthos in mangroves.

Environmental factors such as temperature, sediment composition, and inundation are the main factors influencing the distribution of faunal communities in tropical mangroves. Salinity is one of the important key factors which determine the composition of biological component in the marine environment. The fluctuations in salinity affect the biological characteristics of the environment. The present study did not show characteristic relationship between salinity and macrofaunal distribution; however, soil salinity showed significant negative correlation with species evenness $(r=-0.999 ; \mathrm{p}<0.05)$ at station I (Table 2) and $(\mathrm{r}=-0.960 ; \mathrm{p}<0.05)$ at station III (Table 3$)$. This means that the fluctuation of salinity in riverine and island mangroves have profound influence on the species evenness. Reid GK [38] remarks that the momentary salinity may be regarded as a function of the quantity and quality of inflowing and out flowing waters, rainfall, and evaporations since these factors may vary with seasons (in some instants rather drastically).

In the present investigation, dissolved oxygen was high during the monsoon season at all sites, which might be due to the cumulative effect of higher wind velocity coupled with heavy rainfall and the resultant freshwater mixing. Relatively lower values were observed during summer; this may be due to the increased surface water temperature which reduces the dissolvation of $\mathrm{O}_{2}$ in the coastal waters. It is well known that temperature and salinity affect the dissolution of oxygen [39]. Hydrogen ion concentration $(\mathrm{pH})$ in surface waters remained alkaline at all sites throughout the study period with the maximum value during summer seasons and the minimum during the monsoon. However, the present study did not find a characteristic relationship between $\mathrm{pH}$, salinity, and temperature and macrobenthic fauna, confirming that the fauna of independent mangrove system requires specific environmental characters.

\begin{tabular}{|c|c|c|c|c|c|c|c|c|c|c|c|c|c|}
\hline & ST & SSA & SPH & STN & STP & STOC & Sand & Silt & Clay & Density & Diversity & Richness & Evenness \\
\hline ST & 1 & & & & & & & & & & & & \\
\hline SSA & 0.986091 & 1 & & & & & & & & & & & \\
\hline $\mathrm{SPH}$ & 0.991117 & 0.96058 & 1 & & & & & & & & & & \\
\hline STN & -0.99501 & -0.97095 & -0.98555 & 1 & & & & & & & & & \\
\hline STP & -0.985 & -0.99344 & -0.9533 & 0.980507 & 1 & & & & & & & & \\
\hline STOC & -0.96459 & -0.96499 & -0.96937 & 0.935008 & 0.933743 & 1 & & & & & & & \\
\hline Sand & -0.77014 & -0.86224 & -0.68744 & 0.740486 & 0.858074 & 0.755322 & 1 & & & & & & \\
\hline Silt & 0.799819 & 0.882087 & 0.717767 & -0.77786 & -0.88583 & -0.76537 & -0.99642 & 1 & & & & & \\
\hline Clay & -0.82504 & -0.89623 & -0.74431 & 0.811411 & 0.908346 & 0.770039 & 0.984813 & -0.99597 & 1 & & & & \\
\hline Density & 0.940327 & 0.875838 & 0.975454 & -0.94351 & -0.86945 & -0.91682 & -0.51077 & 0.548353 & -0.58393 & 1 & & & \\
\hline Diversity & 0.946937 & 0.881975 & 0.976773 & -0.95518 & -0.88256 & -0.90725 & -0.52424 & 0.565644 & -0.60513 & 0.997993 & 1 & & \\
\hline Richness & 0.940199 & 0.872801 & 0.973116 & -0.94806 & -0.87202 & -0.90377 & -0.50697 & 0.548085 & -0.58741 & 0.998796 & 0.999715 & 1 & \\
\hline Evenness & -0.99956 & -0.98092 & -0.99279 & 0.997003 & 0.981719 & 0.959627 & 0.753535 & -0.7853 & 0.812843 & -0.94748 & -0.9547 & -0.94825 & 1 \\
\hline
\end{tabular}

Table 2: Simple correlation coefficient $(\mathrm{R})$ between macrofaunal and physico-chemical parameters at station I. 
Citation: Thilagavathi B, Varadharajan D, Babu A, Manoharan J, Vijayalakshmi S, et al. (2013) Distribution and Diversity of Macrobenthos in Different Mangrove Ecosystems of Tamil Nadu Coast, India. J Aquac Res Development 4: 199 doi:10.4172/2155-9546.1000199

Page 10 of 12

\begin{tabular}{|c|c|c|c|c|c|c|c|c|c|c|c|c|c|}
\hline & ST & SSA & SPH & STN & STP & STOC & Sand & Silt & Clay & Density & Diversity & Richness & Evenness \\
\hline ST & 1 & & & & & & & & & & & & \\
\hline SSA & 0.867871 & 1 & & & & & & & & & & & \\
\hline $\mathrm{SPH}$ & 0.930644 & 0.913275 & 1 & & & & & & & & & & \\
\hline STN & -0.79303 & -0.9515 & -0.76116 & 1 & & & & & & & & & \\
\hline STP & -0.84562 & -0.93824 & -0.77308 & 0.990798 & 1 & & & & & & & & \\
\hline STOC & -0.9414 & -0.95002 & -0.87191 & 0.950075 & 0.975984 & 1 & & & & & & & \\
\hline Sand & -0.99612 & -0.86326 & -0.90031 & 0.815045 & 0.871225 & 0.955119 & 1 & & & & & & \\
\hline Silt & 0.838626 & 0.800743 & 0.967909 & -0.58521 & -0.59109 & -0.72117 & -0.78889 & 1 & & & & & \\
\hline Clay & 0.448426 & 0.286066 & 0.11425 & -0.50688 & -0.58958 & -0.54493 & -0.52365 & -0.11045 & 1 & & & & \\
\hline Density & 0.983487 & 0.935874 & 0.97216 & -0.84591 & -0.8751 & -0.95531 & -0.97287 & 0.887245 & 0.343411 & 1 & & & \\
\hline Diversity & 0.985594 & 0.933212 & 0.931586 & -0.88376 & -0.91955 & -0.98285 & -0.98698 & 0.81616 & 0.464778 & 0.990661 & 1 & & \\
\hline Richness & 0.9946 & 0.913559 & 0.940589 & -0.84912 & -0.89028 & -0.968 & -0.99243 & 0.83739 & 0.444172 & 0.993375 & 0.997568 & 1 & \\
\hline Evenness & -0.04257 & -0.36494 & 0.043396 & 0.608955 & 0.562666 & 0.37067 & 0.106685 & 0.260725 & -0.53398 & -0.09677 & -0.19314 & -0.12586 & 1 \\
\hline
\end{tabular}

Table 3: Simple correlation coefficient $(R)$ between macrofaunal and physico-chemical parameters at station II.

\begin{tabular}{|c|c|c|c|c|c|c|c|c|c|c|c|c|c|}
\hline & ST & SSA & SPH & STN & STP & STOC & Sand & Silt & Clay & Density & Diversity & Richness & Evenness \\
\hline ST & 1 & & & & & & & & & & & & \\
\hline SSA & 0.820628 & 1 & & & & & & & & & & & \\
\hline SPH & 0.955647 & 0.952424 & 1 & & & & & & & & & & \\
\hline STN & -0.88275 & -0.98094 & -0.97719 & 1 & & & & & & & & & \\
\hline STP & -0.84942 & -0.96033 & -0.95008 & 0.992338 & 1 & & & & & & & & \\
\hline STOC & -0.6327 & -0.7759 & -0.74357 & 0.847665 & 0.906718 & 1 & & & & & & & \\
\hline Sand & -0.92639 & -0.9451 & -0.97847 & 0.936259 & 0.886342 & 0.609879 & 1 & & & & & & \\
\hline Silt & 0.880526 & 0.45549 & 0.704752 & -0.57842 & -0.55291 & -0.39693 & -0.6493 & 1 & & & & & \\
\hline Clay & 0.738683 & 0.957966 & 0.884171 & -0.89031 & -0.84038 & -0.57064 & -0.93638 & 0.341052 & 1 & & & & \\
\hline Density & 0.890673 & 0.96105 & 0.967448 & -0.93775 & -0.88778 & -0.61132 & -0.99606 & 0.579391 & 0.963771 & 1 & & & \\
\hline Diversity & 0.827154 & 0.963641 & 0.934409 & -0.91792 & -0.86545 & -0.58353 & -0.97754 & 0.474882 & 0.989115 & 0.992222 & 1 & & \\
\hline Richness & 0.82776 & 0.957085 & 0.931135 & -0.91005 & -0.85497 & -0.56534 & -0.97809 & 0.479554 & 0.987637 & 0.99215 & 0.99971 & 1 & \\
\hline Evenness & -0.83512 & -0.96094 & -0.94285 & 0.9904 & 0.999639 & 0.911869 & 0.878709 & -0.53079 & -0.84115 & -0.88224 & -0.86254 & -0.85163 & 1 \\
\hline
\end{tabular}

Table 4: Simple correlation coefficient $(\mathrm{R})$ between macrofaunal and physico-chemical parameters at station III.

Sediment texture plays an important role in the ecology of benthic invertebrates $[40,41]$. The pelagic larvae of macrobenthic organisms before finally settling down at the bottom have to cross many barriers, and each type of bottom deposit will attract a very limited and selected set of species [42]. A common concept in benthic animal-sediment relation is that the feeding type of the infauna is in one way correlated to the sediments [43]. Deposit or detritus feeders constitute an important and often dominating part of macrobenthic invertebrates [44]. Sediment character has been identified as one of the driving forces in determining the macrofaunal communities. At station I, species diversity is negatively correlated with sand $(r=-0.986)$ while in station II and III, positive correlation is obtained between density and silt $(\mathrm{r}=0.984$ and $\mathrm{r}=0.887)$ at $\mathrm{p}<0.05$ level (Table 4$)$.

This indicates that availability of silty soil sustains to macrofaunal diversity and density, while sand dominance will reduce the macrofaunal population. Clayey silt substrate is always known to support epifauna $[45,46]$. Food supply seldom acts as a limiting factor in the seasonal abundance of macrobenthos [47]. Organic nutrients enhance the growth of different types of algae that provide food resources for benthos [48]. In the present study, the higher density macrobenthos is observed during summer season. Higher organic matter gets deposited during the post-monsoon season in the mangrove areas. It would be converted into available organic carbon by various fungal and bacterial sources, which in turn increase the macrobenthic forms especially polychaetes [49]. High organic carbon induced abundance of macrofauna in Coleroon estuary [50]. This confirmed that the abundance of benthic fauna is highly related to organic carbon.
To find out a clear picture of species diversity and distribution, various univariate and multivariate analyses have been carried out and results are discussed. Individual species is a simple and useful measure of the biological system. The species individual diversity in the present study registered a wide fluctuation between 4.311 (monsoon) and 5.167 (summer) among stations and seasons. The lower species diversity was recorded during monsoon and higher diversity values during summer in the study area. This is in conformity with the earlier observations made in Vellar [51] and Coleroon estuaries [31]. Moreover, Pearson $\mathrm{TH}$ and Rosenberg $\mathrm{R}$ [52] proposed that the use of diversity indices is advantageous for the description of fauna at different stages in succession. In the present study, negative correlation is obtained between species richness and diversity at stations I ( $\mathrm{r}=-0.999)$, II $(\mathrm{r}=-$ $0.997)$ and III $(\mathrm{r}=-0.999)$ at $\mathrm{p}<0.05$ level.

All the nutrients (TN, TP and TOC) are found enriched in the sediment during monsoon. These nutrients might have reached the benthic realm through food web during summer and pre-monsoon seasons. The species richness of benthic macrofauna was found maximum during the summer season (32.70). A similar observation was reported by Kumar RS [37] in Cochin backwaters. The low richness was recorded during monsoon (16.69) might be due to the high freshwater inflow with low saline conditions, which in turn affect the distribution of benthos, particularly the polychaetes. Maximum diversity and richness recorded during summer at the all sites might be due to stable and optimum environmental factor such as salinity, which plays an important role in faunal distribution and abundance. Shannon diversity was exceptionally high and it was in the range of 4.311-5.167. The minimum (4.311) and maximum (5.167) was recorded at station II 
Citation: Thilagavathi B, Varadharajan D, Babu A, Manoharan J, Vijayalakshmi S, et al. (2013) Distribution and Diversity of Macrobenthos in Different Mangrove Ecosystems of Tamil Nadu Coast, India. J Aquac Res Development 4: 199 doi:10.4172/2155-9546.1000199

during the monsoon season and at station I during the summer season, respectively. Shannon diversity in the present study was considered to be good and the range recorded in the present study vouchsafes for the healthy nature of mangrove ecosystem. Similar seasonal pattern is evident from the view of Kundu et al. [53].

Species area plots used to show the cumulative number of different species observed as each new sample is added. The advantage of plotting this technique is to predict the total number of stations to be sampled for getting the maximum number of species in a station. The present study revealed that 12 times sampling during various seasons is enough to get all the species in the study areas. The dendrograms derived in the present study showed clustering of stations and gradual change in species composition from the island mangrove ecosystem towards riverine mangrove ecosystem. This means that a certain level of similarity prevails in faunal diversity in developing and island mangroves than in the riverine mangrove ecosystem. Derived multidimensional scaling (MDS) ordination reveals the same grouping of stations as in the cluster analysis. The stress values found in the MDS configuration is low (0.11), indicating good representation of the interrelationship between the macrofauna of each station. Benthic population density $(\mathrm{N})$ is positively correlated with sediment temperature $(\mathrm{r}=0.940)$, salinity $(\mathrm{r}=0.875), \mathrm{Ph}(\mathrm{r}=0.975)$, Silt $(\mathrm{r}=0.548)$ in station I. In station II, benthic population density is positively correlated with sediment temperature $(\mathrm{r}=0.983)$, salinity $(\mathrm{r}=0.935), \mathrm{pH}(\mathrm{r}=0.972)$ and silt $(\mathrm{r}=0.887)$. In station III, sediment temperature $(\mathrm{r}=0.890)$, sediment salinity $(\mathrm{r}=0.961), \mathrm{pH}$ $(\mathrm{r}=0.967)$ and silt $(\mathrm{r}=0.579)$ are positively correlated with benthic population at $\mathrm{p}<0.05$ level.

\section{Conclusion}

Among the three ecosystems, riverine mangrove (station I) ecosystem is more pristine in nature than the developing and island mangrove ecosystems. Benthic macrofauna species assemblage is comparatively higher in station I than in stations II and III. Analysis of data undertaken with predictable like line Shannon diversity, Simpson richness, and recently introduced diversity indices such as taxonomic diversity index and total phylogenetic index clearly opined that healthy nature of the mangrove ecosystem. From the present study, it could be concluded that the hydrography, nutrients, and sediment texture are the major factors responsible for fluctuation in benthic macrofaunal assemblages in the study area.

\section{References}

1. Manson FJ, Loneragan NR, Skilleter GA, Phinn SR (2005) An Evaluation of the Evidence for Linkages between Mangroves and Fisheries: A Synthesis of the Literature and Identification of Research Directions. Oceanogr Mar Biol Annul Rev 43: 485-515

2. Krom MD, Berner RA (1980) Adsorption of phosphate in anoxic marine sediments. Limnol Oceanogr 25: 797-806.

3. Srilatha G, Thilagavathi B, Varadharajan D (2012) Studies on the physicochemical status of muthupettai mangrove, south east coast of India. Advan Appl Sci Res 3: 201-207.

4. Raveenthiranath N (1990) Ecology of Macrobenthos in and around Mahandrapalli Region of Coleroon Estuary, Southeast coast of India. Ph.D. thesis, Annamalai University, India, 231.

5. Kathiresan K (2000) A review of studies on pichavaram mangroves, southeast India. Hydrobiologia 430: 185-205.

6. Kathiresan K, Quasim SZ (2005) Biodiversity of Mangrove Ecosystems New Delhi: Hindustan Publishing Corporation. p 251.

7. Warren JH, Underwood AJ (1986) Effects of burrowing crabs on the topography of mangroves swamps in New South Wales. J Exp Mar Biol Ecol 102: 223-235.

8. Smith TJ, Boto KG, Frusher SD, Giddins RL (1991) Keystone species and mangrove forest dynamics: the influence of burrowing by crabs on soil nutrient status and forest productivity. Estuar Coast Shelf S 33: 419-432.

9. Thilagavathi B, Varadharajan D, Manoharan J, Vijayalakshmi S Balasubramanian T (2012) Taxonomy and Distribution of Benthic Foraminifera from the Sediment of Palk Strait, South East Coast of India. Int J Pharma Bio Arch 3: 1129-1136

10. Manoharan J, Varadharajan D, Thilagavathi B, Priyadharsini S (2011) Biodiversity and abundance of benthos along the South East Coast of India. Advan Appl Sci Res 2: 554-562.

11. Oswin SD (1998) Biodiversity of the Muthupet Mangroves, South East Coast of India. Sesaiyana 6: 9-11.

12. Oswin SD, Kannadasan $P$ (1998) Mangrove insects and spiders of Muthupet, Tamil Nadu. Environ Ecol 16: 932-936.

13. Strickland JDH, Parsons TR (1972) A Practical Handbook of Seawater Analysis. Fish Res Board Canada, 310

14. Jackson ML (1958) Soil Chemical Analysis. New Delhi: Prentice Hall.

15. Krumbein WC, Pettijohn FJ (1938) Manual of Sedimentary Petrography. New York: Appleton Century Crofts.

16. Fauvel P (1953) The fauna of India including Pakistan, Ceylon, Burma and Malaya, Annelida Polychaeta. Allahabad: The Indian Press.

17. Day JH (1967) A Monograph on the Polychaeta of Southern Africa, Part (Errantia) \& II (Sedentaria). London: Trustees of the British Museum (Natural History).

18. Subba Rao NV, Surya Rao KV, Maitra S (1991) Marine mollusks, State Fauna Series, Fauna of Orissa, Kolkata. Zool Sur India 1: 1-175.

19. Gray J (1974) Animal-sediment relationships. Oceanog Mar Biol Annul Rev 12 223-261.

20. Rhoads D (1974) Organism-sediment relations on the muddy sea floor Oceanog Mar Biol 12: 263-300.

21. Snelgrove PVR, Butman CA (1994) Animal Sediment Relationships Revisited Cause Versus Effect. Oceanog Mar Biol 32: 111-177.

22. Aller JY, Woodin SA, Aller RC (2001) Organism Sediment Interactions. Columbia: University of South Carolina Press.

23. Reish DJ (1965) The effect of fresh-water runoff on a population of estuarine polychaetous annelids. Bull Southern Calif Acad Sci 64: 111-119.

24. Postma H (1954) Hydrography of the Dutch Wadden Sea. Thesis Groningen. Arch. Neerland. Zool X, 4e Livraison 106.

25. Schelske CL, Odum EP (1961) Mechanisms Maintaining High Productivity In Georgia Estuaries. In: Proceed Gulf Caribbean Fish Ins 14th Annul Ses 75-80.

26. Parulekar AH, Waugh (1975) Benthos studies in Goa estuaries III. Annual Cycle of Macrofaunal Distributional Production and Trophic Relations. Indian J Mar Sci 1: 189-200.

27. Parulekar AH, Ansari ZA (1986) Spatial and temporal changes in benthic macrofauna from mandovi \& zuari estuaries of Goa, West coast of India. Indian J Mar Sci 15: 223-229.

28. Harikantra SN, Nair A, Ansari ZA, Parulekar AH (1980) Benthos of Shelf Region along the West Coast of India. Indian J Mar Pollu Bull 14: 41-46.

29. Sankar G (1998) Studies on the Hydrobiology, Benthic Ecology and Fisheries of Muthupet Lagoon. Ph.D. Thesis, Annamalai University, India, 105.

30. Sunil Kumar (1995) Macrobenthos in mangrove ecosystem of Cochin backwater, Kerala (South west coast of India). Indian J Mar Sci 24: 56-61.

31. Prabha Devi L (1994) Ecology of Coleroon estuary, Studies on Benthic Fauna J Mar Biol Ass India 36: 260-266.

32. Ansari ZA, Ingole BS, Banerjee G, Parulekar AH (1986) Spatial And Tempora Changes In Benthic Macrofauna From Mandovi \& Zuari Estuaries Of Goa, West coast of India. Indian J Mar Sci 15: 223-229.

33. Athalye RP, Gokhale KS (1998) Macrobenthos from the mudflats of Thane Creek, Maharashtra, India. J Bombay Nat His Soc 95: 258-266.

34. Mishra A, Choudhury (1985) Polychaetes Annelids from the Mangrove Swamps of Sundarbans, India. Proc Nat Symp Biol Util Conser Mang 448-452. 
Citation: Thilagavathi B, Varadharajan D, Babu A, Manoharan J, Vijayalakshmi S, et al. (2013) Distribution and Diversity of Macrobenthos in Different Mangrove Ecosystems of Tamil Nadu Coast, India. J Aquac Res Development 4: 199 doi:10.4172/2155-9546.1000199

35. Kathiresan K, Rajendran N, Palaniselvam V (2000) Growth of Rhizophora apiculata in degraded areas of Ariyankuppam estuary along Pondicherry coastline, southeast coast of India. Indian J Mar Sci 29: 86-88.

36. Mohammed SZ (1995) Observation on the benthic macrofauna of the soft sediment on western side of the Arabian Gulf (ROPME sea area) with respect to 1991 Gulf War oil spill. Indian J Mar Sci 24: 147-152.

37. Kumar RS (2001) Macrobenthos in the Mangrove Ecosystem of Cochin backwaters, Kerala, southwest coast of India. Indian J Mar Sci 24: 56-61.

38. Reid GK (1961) Ecology of Inland Waters and Estuaries. New York: Reinhold.

39. Viijayakumar S, Rajesh KM, Mridula RM, Hariharan V (2000) Seasonal Distribution And Behaviour of Nutrients With Reference to Tidal Rhythm in the Mulki estuary, Southwest coast of India. J Mar Biol Ass India 42: 21-31.

40. Sanders HL (1958) Benthic studies in Buzzards Bay. I. Animal-Sediment Relationships. Limnol Oceanog 3: 245-258.

41. Maurer D, Vargas JA (1984) Diversity of soft-bottom benthos in a tropical estuary: Gulf of Nicoya, Costa Rica. Mar Biol 81: 97-106.

42. Thorson G (1966) Some factors Influencing the Requirement and Establishment of Marine Benthic Communities. J Sea Res 3: 267-293.

43. Bloom SA, Simon JL, Hunter VD (1972) Animal-sediment relations and community analysis of a Florida estuary. Mar Biol 13: 43-56.

44. Fenchel T, Kofoed LH, Lappalainen A (1975) Particle size-selection of two deposit feeders: the amphipod Corophium volututor and the prosobranch Hydrobiu ulvae. Mar Biol 30: 119-128.

45. Kondala Rao B, Ramanamurthy KV (1988) Ecology of Intertidal Meiofauna of the Kakin Bay (Gautam-Godavari Estuarine System), east coast of India. Indian J Mar Sci 17: 40-47.

46. Goldin Q, Mishra V, Ullal V, Athalye AR, Gokhale KS (1996) Meiobenthos of mangrove mudflats from shallow region of Thane creek, central west coast of India. Indian J Mar Sci 25: 137-141.

47. Qasim SZ (1970) Some problems related to the food chain in a tropical estuary In: Mar food chains, J.H. Steele (Ed.). University of California Press, Los Angeles, USA, 45-51.

48. Hearld CL, Odum EP (1970) Mechanisms Maintaining High Productivity in Georgia estuaries. Proceed Gulf Caribbean Fish Ins 14th Annul Ses 75-80.

49. Sunilkumar R, Antony A (1994) Preliminary Studies on the Polychaete Fauna of the Mangrove Areas of Cochin. In: Proceedings of the 6th Kerala Science Congress, Thiruvanthapuram, Kerala, R. Ravikumar (Eds), State Commit Sc Technol Environ 74-77.

50. Prabha Devi L, Ayyakkannu K (1991) Ecology of benthic macrofauna in Cuddalore-Uppanar backwater, southeast Coast of India. Indian J Mar Sci 20: 200-203.

51. Chandran R (1987) Hydrobiological Studies in the Gradient Zone of the Vellar estuary. IV. Benthic Fauna. Mahasagar Bull Nat Ins Oceanog 20: 1-13.

52. Pearson TH, Rosenberg $R$ (1978) Macrobenthos secession in relation to organic enrichment and pollution of the marine environment. Oceanog Mar Biol Annul Rev 16: 229-234.

53. Kundu S, Mondal N, Lyla PS, Ajmal Khan S (2010) Biodiversity and seasonal variation of macro-benthic infaunal community in the inshore waters of Parangipettai Coast. Environ Monitor Asses 163: 67-79. 